\title{
The ALE-method with triangular elements: direct convection of integration point values
}

\author{
M.J. van Haaren H.C. Stoker A.H. van den Boogaard \\ J. Huétink \\ University of Twente, Fac. Mechanical Engineering \\ P.O. Box 217, 7500 AE Enschede, The Netherlands
}

November 16, 1999

\begin{abstract}
Correspondence to: A.H. van den Boogaard, University of Twente, Fac. Mechanical Engineering, P.O. Box 217, 7500 AE Enschede, The Netherlands

E-mail: A.H.vandenBoogaard@wb.utwente.nl

The research, described in this paper was sponsored by Stichting Werkplaatstechniek (SWT).
\end{abstract}

\begin{abstract}
The Arbitrary Lagrangian-Eulerian (ALE) Finite Element Method is applied to the simulation of forming processes where material is highly deformed. Here, the split formulation is used: a Lagrangian step is done with an implicit finite element formulation, followed by an explicit (purely convective) Eulerian step. The purpose of this study is to investigate the Eulerian step for quadratic triangular elements. To solve the convection equation for integration point values, a new method inspired by Van Leer [1] is constructed. The new method is based on direct convection of integration point values without intervention of nodal point values.

The Molenkamp test and a so-called block test were executed to check the performance and stability of the convection scheme. From these tests it is concluded that the new convection scheme shows accurate results. The scheme is extended to an ALE-algorithm. An extrusion process was simulated to test the applicability of the scheme to engineering problems. It is concluded that direct convection of integration point values with the presented algorithm leads to accurate results and that it can be applied to ALE-simulations.
\end{abstract}

KEY WORDS: convection schemes, unstructured grids, plasticity, forming processes 


\section{Introduction}

In solid mechanics usually Updated Lagrangian formulations are applied for solving large inelastic deformation problems. Advantages of these formulations are that history dependent properties, as for example strain hardening, and free surfaces can be taken into account easily. However, in forming processes the element mesh becomes too distorted after a number of steps which leads to inaccuracy and finally to a break down of the analysis. Therefore, a Lagrangian formulation is not convenient to simulate forming processes as extrusion and rolling in which material is highly deformed.

On the other hand, problems in fluid dynamics are commonly formulated in Eulerian coordinates. The material flows through the mesh. The element mesh is not distorted, so that the calculation can be proceeded infinitely. However, standard Eulerian formulations can not describe free surfaces and it is difficult to treat history dependent properties. For forming processes description of free surfaces and history dependence are necessary to obtain correct results.

Arbitrary Lagrangian-Eulerian (ALE) formulations combine the advantages of the Lagrangian and the Eulerian method. In literature several mixed Euler-Lagrange formulations can be found, see Liu et al. [2], Benson [3], Baaijens [4], Huétink [5], Donea [6], Hughes et al. [7]. These formulations can be divided into two groups, respectively coupled and split formulations. In the framework of this research we use the split formulation. In the Lagrangian step the material increment of the variables is determined with an implicit finite element formulation. The Eulerian step can be considered as a remap from one element mesh to another element mesh. Since the topology of the mesh does not change, the Eulerian step can be represented by the convection equation (see section 2).

The purpose of this research is to investigate the Eulerian part of the calculation for quadratic triangular elements with three integration points. These elements are known to give accurate results and they are easy to use for the construction of complex finite element meshes.

There are several methods dealing with the convection problem in an ALE-analysis, see Huerta et al. [8], Baaijens [9], Stoker [10] and Benson [3]. Most methods for solving the convection equation use a finite volume formulation with a cell-centered approach, see for example Tamamidis [11] for triangular elements. In our case the initial situation is defined in the three integration points. A cell-centered approach may lead to loss of accuracy. If no variation of the convected quantity over an element is taken into account, it can be derived that the solution is first-order accurate. For second-order accuracy the gradient of the convected quantity has to be dealt with. First-order schemes are known to be diffusive, however second-order ones could introduce spurious oscillations without some form of limiting $[11,12]$. Inspired by Van Leer [1], we introduce an integration point based convection scheme, which is second-order accurate (see section 3). As opposed to Van der Helm [13] this scheme can be applied to structured grids as well as unstructured grids.

In order to check the performance and stability of the new scheme, the Molenkamp test and a more severe block test were performed. Results can be found in section 4 . 
For smooth problems with grid-aligned flow a first order upwind scheme might be satisfactory. But, for instance visco-elastic problems [14] or problems with unstructured grids, where no grid-alignment can be distinguished, require a more accurate convection scheme. Therefore, the scheme is also applied to an ALE-formulation and in section 5 results of an extrusion process are shown as an example. Finally, in section 6 some conclusions are given.

\section{ALE-formulation}

The Finite Element Method is used to solve the mechanical equilibrium of a structure. This equilibrium can be written in integral form through the weak formulation:

$$
\begin{array}{rlrl}
\delta W & =\int_{\Omega} \delta \mathbf{d}: \boldsymbol{\sigma} \mathrm{d} \Omega-\int_{\Omega} \mathbf{f} \cdot \delta \mathbf{v} \mathrm{d} \Omega-\int_{\partial \Omega} \mathbf{t} \cdot \delta \mathbf{v} \mathrm{d} \Gamma=0 & \forall \delta \mathbf{v} \\
\delta \mathbf{d} & =\frac{1}{2}(\delta \mathbf{v} \overleftarrow{\nabla}+\vec{\nabla} \delta \mathbf{v})
\end{array}
$$

where $\Omega$ is the current volume of the structure and $\partial \Omega$ is the boundary of $\Omega . \boldsymbol{\sigma}$ is the Cauchy stress tensor, the vector $\mathbf{f}$ represents the body forces, the vector $\mathbf{t}$ represents the surface forces, and $\delta \mathbf{v}$ is denoted as the virtual velocity. In this article we use a rigid-plastic material model to calculate the stress tensor $\boldsymbol{\sigma}$, but the method works for an elasto-plastic material model too [15, 16].

Since plastic deformation processes are path dependent, an incremental procedure is used. The new state after an increment is only calculated for a finite number of grid points of the mesh: the nodal points (for displacement, temperature) and the integration points (for stresses, strains). In the Updated Lagrangian method these grid points are connected to the material, and the coordinates of these points are updated before the next increment is started. In section 1 the disadvantages of the Updated Lagrangian method in calculations with large deformations are reviewed. In the ALE-method the material displacement increments are uncoupled from the grid point displacement increments. This leads to an apparent material flow through the elements. The displacement increments of the grid points can be chosen more or less freely, however, there are some restrictions. A point which lies for example at a free surface, must remain on that surface, but not necessarily at the same material particle. In our case the variables in the grid points are updated after convergence of the Lagrangian part of the calculation.

Uncoupling of the material and grid point displacement implies that in addition to the incremental calculation in the Lagrangian step, convection in the Eulerian step must be taken into account. This can be represented by the convection equation:

$$
\begin{cases}\frac{\partial q}{\partial t}+\mathbf{v}^{c} \cdot \frac{\partial q}{\partial \mathbf{x}}=0 & \mathbf{x} \in \Omega, t \in \mathbb{R}^{+} \\ q(\mathbf{x}, 0)=q_{0}(\mathbf{x}) & \mathbf{x} \in \Omega\end{cases}
$$


The convected quantity is denoted by $q$. In our examples this represents the state variables e.g. the equivalent plastic strain. For quasi static processes, these are the only quantities to be convected. If inertia effects are to be taken into account, the nodal velocities must also be convected. Note that in a split ALE algorithm, the 'convection' takes place in a 'frozen' time increment. This paper only describes the convection of the discontinuous integration point data for quasi static processes.

The relative velocity between the material and grid, the convective velocity, is given by $\mathbf{v}^{c}=\mathbf{v}^{g}-\mathbf{v}^{m}$ where $\mathbf{v}^{g}$ and $\mathbf{v}^{m}$ represent the grid (mesh) velocity and the material velocity respectively. The material velocity is known from the Lagrangian step and the grid velocity is determined in a mesh management procedure [17], which is performed before convection can be processed. Consequently, prior to the Eulerian step the convective velocity is known.

The initial distribution $q_{0}$ is defined by the situation after the Lagrangian part of the calculation. The scalar function $q$ represents history dependent variables known in the integration points of the element. Hence, in the convection step the integration point values have to be updated. In a Finite Element simulation that uses an ALE-formulation, a convenient convection scheme is needed.

A convection scheme has to satisfy a number of requirements. The convection scheme has to be accurate, otherwise the accuracy of the Lagrangian step is lost. Furthermore, the scheme must be stable, which means that after the remap the value of the convected quantity $q$ remains bounded, and monotone, so that no spurious oscillations are introduced. Other important requirements are consistency, conservativity and constancy. Consistency requires that the scalar function $q$ does not change in the limit case that $\mathbf{v}^{c}$ is zero. Conservativity means that the integral of $q$ over the domain does not change and constancy is the property that an initially uniform field remains uniform in case of arbitrary relative grid displacements. Finally, an efficient scheme is required, since the remap is carried out every time step.

\section{Convection}

As already mentioned in section 1 the gradient of the convected quantity over an element must be taken into account to reach second-order accuracy. Since the initial distribution is given in the integration points, it is most obvious to calculate this gradient from integration point values. Van Leer [1] describes a method to solve the convection equation on onedimensional grids. This method starts from a piece-wise approximation of the initial-value distribution by simple basic functions, as for example the mean value and the local gradient in an element. Overall a discontinuous distribution results, which is convected explicitly and then remapped piece-wise in terms of the same basic functions. Below, this method is applied on two-dimensional grids of triangles. Convection in an arbitrary direction is processed in two perpendicular directions ( $x$ - and $y$-direction) simultaneously.

The method is split into the following three steps. 


\section{Step 1}

Since $q$ within one element is only known in three discrete points (the integration points), we construct a linear distribution $\bar{q}_{i}\left(x, y, t_{0}\right)$ per element $i$. Therefore, the following values are calculated for each element $i$ :

- Mean value in the central point: $\bar{q}_{i}^{0}$.

- Gradient in $x$-direction: $\frac{\overrightarrow{\partial q}_{i}^{0}}{\partial x}$.

- Gradient in $y$-direction: $\frac{\overrightarrow{\partial q}_{i}^{0}}{\partial y}$.

With these values the linear distribution $\bar{q}_{i}$ is given by:

$$
\begin{aligned}
\bar{q}_{i}\left(x, y, t_{0}\right)= & \bar{q}_{i}^{0}+\frac{\overline{\partial q}_{i}^{0}}{\partial x}\left(x-\bar{x}_{i}\right)+\frac{\overline{\partial q}_{i}^{0}}{\partial y}\left(x-\bar{y}_{i}\right) \\
& (x, y) \in \text { element } i
\end{aligned}
$$

where $\left(\bar{x}_{i}, \bar{y}_{i}\right)$ is the central point. The functions $\bar{q}_{i}\left(x, y, t_{0}\right)$ for all elements together form the total approximate distribution $\bar{q}\left(x, y, t_{0}\right)$ which is discontinuous over the element boundaries. Figure 1a shows $\bar{q}\left(x, y, t_{0}\right)$ for a one-dimensional situation which is easier to interpret than a two-dimensional situation.

For elements with more than three integration points, this kind of linear distributions becomes over determined. In that case it is most obvious to construct a linear distribution using a least square fit of the integration point values, see e.g. Stoker [16] where this has been performed for quadrilateral elements with four integration points.

\section{Step 2}

Starting from the approximate distribution $\bar{q}\left(x, y, t_{0}\right)$, Equation (3) is integrated over a finite time step $\Delta t$. This is achieved by shifting $\bar{q}\left(x, y, t_{0}\right)$ over a distance $\mathbf{v}^{c} \Delta t$ :

$$
\begin{aligned}
& \bar{q}\left(x, y, t_{1}\right)=\bar{q}\left(x-u \Delta t, y-v \Delta t, t_{0}\right) \\
& t_{1}=t_{0}+\Delta t
\end{aligned}
$$

where $u$ and $v$ are the components of the convective velocity $\mathbf{v}^{c}$ in $x$ - and $y$-direction respectively. This leads to a shifted piece-wise linear distribution where discontinuities appear inside the elements as demonstrated in Figure 1b. For each element $i$ an updated linear distribution $\bar{q}_{i}\left(x, y, t_{1}\right)$ as shown in Figure 1c must be constructed. Therefore, it is needed to determine updated mean values $\bar{q}_{i}^{1}$, and updated gradients $\frac{\bar{q}_{i}^{1}}{\partial x}$ and $\frac{\overline{\partial q}_{i}^{1}}{\partial y}$ from the shifted distribution at $t=t_{1}$. This is discussed in the next two sections.

\section{Step 3}

New integration point values are calculated from the new approximate distribution $\bar{q}\left(x, y, t_{1}\right)$. 


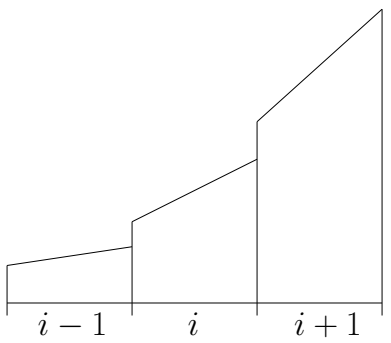

(a)

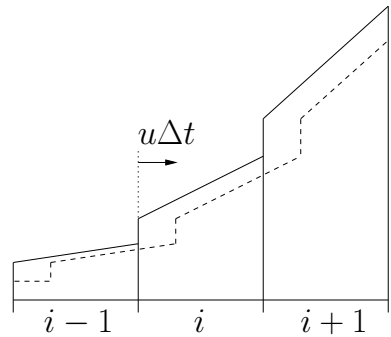

(b)

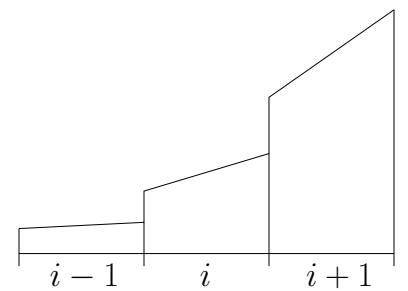

(c)

Figure 1: Shifting the piece-wise linear distribution for a one-dimensional situation. (a) The approximate distribution $\bar{q}\left(x, t_{0}\right)$. (b) The approximate distribution $\bar{q}\left(x, t_{0}\right)$ (solid line) and the shifted distribution $\bar{q}\left(x-u \Delta t, t_{0}\right)$ (dashed line). (c) The updated approximate distribution $\bar{q}\left(x, t_{1}\right)$.

The updated linear distribution $\bar{q}_{i}\left(x, y, t_{1}\right)$ for element $i$ is constructed with the values $\bar{q}_{i}^{1}$, $\frac{\overline{\partial q}_{i}^{1}}{\partial x}$ and $\frac{\overline{\partial q}_{i}^{1}}{\partial y}$ and is given by:

$$
\begin{aligned}
\bar{q}_{i}\left(x, y, t_{1}\right)= & \bar{q}_{i}^{1}+\frac{\overline{\partial q}_{i}^{1}}{\partial x}\left(x-\bar{x}_{i}\right)+\frac{\overline{\partial q}_{i}^{1}}{\partial y}\left(x-\bar{y}_{i}\right) \\
& (x, y) \in \text { element } i
\end{aligned}
$$

The described method boils down to update the mean value $\bar{q}_{i}$, and the gradients $\frac{\overline{\partial q}_{i}}{\partial x}$ and $\frac{\overline{\partial q}_{i}}{\partial y}$ for all elements.

\subsection{Determination of the updated mean value}

The mean value is updated on the basis of the following equation:

$$
\int_{A_{i}} \bar{q}\left(x, y, t_{1}\right) \mathrm{d} \Omega=\int_{A_{i}} \bar{q}\left(x-u \Delta t, y-v \Delta t, t_{0}\right) \mathrm{d} \Omega
$$

where $A_{i}$ is the area of element $i$. Since the linear distribution is constructed from the central point of the triangular element (see (4) and (6)), the linear terms of $\bar{q}\left(x, y, t_{1}\right)$ in the left hand side vanish. The right hand side is an integral over a discontinuous distribution (see Figure 1b), but can be approximated quite simply. Equation (7) yields:

$$
A_{i} \bar{q}_{i}^{1}=A_{i} \bar{q}_{i}^{0}-\sum_{k=1}^{3} F_{i, k}^{0}
$$

where $F_{i, k}^{0}$ is the flux 'through' side $k$ of element $i$, calculated as the volume under the shaded part of Figure $2 \mathrm{a}$ or $2 \mathrm{~b}$ dependent on the direction of the normal velocity $v_{n, k}$. The normal velocity $v_{n, k}$ is calculated as the average of the inner products of the velocities in 


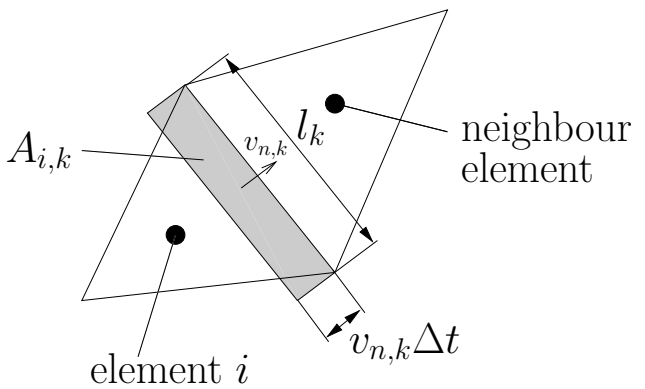

(a)

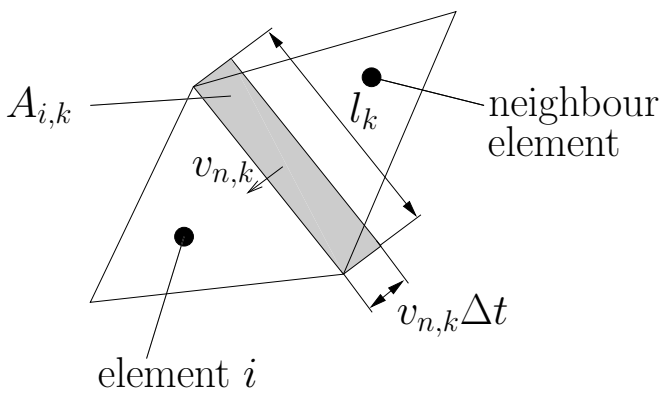

(b)

Figure 2: The flux 'through' side $k$ of element $i$. (a) Outgoing flux. (b) Ingoing flux.

the three nodal points of side $k$ with the outward pointing normal vector $\mathbf{n}_{i, k}$. On the basis of the normal velocity the upwind element is determined. Here, the upwind element is defined as the element from which the information, needed to calculate $F_{i, k}^{0}$, comes. For every side $k$ of the triangle an upwind element must be determined. The upwind element is given by element $i$ itself (Figure 2a) or its neighbour element at side $k$ (Figure 2b). After that, $F_{i, k}^{0}$ is given by the following integral:

$$
\begin{aligned}
F_{i, k}^{0} & =\operatorname{sgn}\left(v_{n, k}\right) \int_{A_{i, k}} \bar{q}_{\text {up }}\left(x, y, t_{0}\right) \mathrm{d} \Omega \\
& =\operatorname{sgn}\left(v_{n, k}\right) \int_{A_{i, k}}\left\{\bar{q}_{\text {up }}^{0}+\frac{\overline{\partial q}_{\text {up }}^{0}}{\partial x}\left(x-\bar{x}_{\text {up }}\right)+\frac{\overline{\partial q}_{\text {up }}^{0}}{\partial y}\left(y-\bar{y}_{\text {up }}\right)\right\} \mathrm{d} \Omega
\end{aligned}
$$

where $A_{i, k}$ is the area of the shaded part in the upwind element. The integral in the right hand side of (9) can be determined in several manners. In this work it was done analytically, as worked out in appendix A, but it can also be done with numerical integration techniques as Gauss-Legendre quadrature [18].

The flux $F_{i, k}^{0}$ gives a positive contribution if the normal velocity $v_{n, k}$ is outward pointing $\left(v_{n, k} \geq 0\right)$ and a negative contribution if the normal velocity is inward pointing $\left(v_{n, k}<0\right)$. Of course, if the flux is an outgoing flux for the element itself, it will be an ingoing flux for the neighbour element as will be observed during an earlier or later stage of the calculation. Hence, conservativity is guaranteed. Since the real trapezoidal fluxes are replaced by rectangles, constancy is only met approximately if the area of the element changes. The solution improves, regarding the constancy requirement, on reduction of the time step. If the change in area is large, a proper correction for the change in area could be performed, but in most practical situations this is not required.

\subsection{Determination of updated gradients}

Updating the gradients $\frac{\overline{\partial q}_{i}^{0}}{\partial x}$ and $\frac{\overline{\partial q}_{i}^{0}}{\partial y}$ is more difficult. Below, two possible methods are discussed. 


\section{Method I}

For the one-dimensional situation Van Leer discusses a method to update the derivative from the shifted values of the approximate function $\bar{q}$ on the edges of an element. Assuming $x_{e}$ the location of an edge, the shifted function value is given by $\bar{q}\left(x_{e}-u \Delta t, t_{0}\right)$.

For two-dimensional elements a similar method is defined. This method uses Gauss' theorem. For an area $\Omega$, bounded by $\partial \Omega$ and $q$ a scalar field, continuously differentiable on $\Omega+\partial \Omega$, holds:

$$
\int_{\Omega} \nabla q \mathrm{~d} \Omega=\oint_{\partial \Omega} q \mathbf{n} \mathrm{d} \Gamma
$$

with $\mathbf{n}$ the outward normal vector. For constant $\nabla q$ this relation yields:

$$
\Rightarrow \nabla q=\frac{1}{\Omega} \oint_{\partial \Omega} q \mathbf{n} \mathrm{d} \Gamma
$$

For a triangle $i(11)$ can be written as:

$$
\overline{\nabla q}_{i}^{1}=\frac{1}{A_{i}} \sum_{k=1}^{3} \mathbf{n}_{i, k} \int_{\partial \Omega_{i, k}} \bar{q}\left(x-u \Delta t, y-v \Delta t, t_{0}\right) \mathrm{d} \Gamma
$$

where $\partial \Omega_{i, k}$ represents edge $k$ of element $i$. The integral in the righthand side of (12) is calculated based on information of the upwind element (see section 3.1). For an arbitrary side $k$ of an arbitrary element $i$ this integral is given by:

$$
\begin{aligned}
\int_{\partial \Omega_{i, k}} \bar{q}\left(x-u \Delta t, y-v \Delta t, t_{0}\right) \mathrm{d} \Gamma & = \\
& \int_{\partial \Omega_{i, k}}\left\{\bar{q}_{\mathrm{up}}^{0}+\frac{\overline{\partial q}_{\mathrm{up}}^{0}}{\partial x}\left(x-u_{\mathrm{up}} \Delta t-\bar{x}_{\mathrm{up}}\right)+\frac{\overline{\partial q}_{\mathrm{up}}^{0}}{\partial y}\left(y-v_{\mathrm{up}} \Delta t-\bar{y}_{\mathrm{up}}\right)\right\} \mathrm{d} \Gamma
\end{aligned}
$$

Breaking up the normal vector $\mathbf{n}_{i, k}$ into the $x$ - and $y$-direction gives a contribution to the gradient in $x$ - and $y$-direction respectively.

Of course, it has to be realized that $\bar{q}\left(x-u \Delta t, y-v \Delta t, t_{0}\right)$ is not continuously differentiable on an element. However, it is assumed that the scalar field $q$ is sufficiently smooth to do no harm to this requirement.

This method has one peculiarity: for vanishingly small $\mathbf{v}^{c} \Delta t$ values it yields a nonvanishing change in the derivatives. This is a consequence of the discontinuities in $\bar{q}\left(x, y, t_{0}\right)$ at the element boundaries. The method tends to annihilate these discontinuities by adjusting the derivatives.

\section{Method II}

Another method that Van Leer discusses in his article is based on holding the first moment 
of $\bar{q}$ constant. For the two-dimensional case with triangles this principle is extended to first moments along the $x$ - and $y$-axis:

$$
\begin{aligned}
\int_{A_{i}} \bar{q}\left(x, y, t_{1}\right)\left(x-\bar{x}_{i}\right) \mathrm{d} \Omega & =\int_{A_{i}} \bar{q}\left(x-u \Delta t, y-v \Delta t, t_{0}\right)\left(x-\bar{x}_{i}\right) \mathrm{d} \Omega \\
\int_{A_{i}} \bar{q}\left(x, y, t_{1}\right)\left(y-\bar{y}_{i}\right) \mathrm{d} \Omega & =\int_{A_{i}} \bar{q}\left(x-u \Delta t, y-v \Delta t, t_{0}\right)\left(y-\bar{y}_{i}\right) \mathrm{d} \Omega
\end{aligned}
$$

This second method coincides with a minimization of the least square error for the new distribution. In case of triangles the least square error is taken over an element and is given by:

$$
\Phi=\int_{A_{i}}\left(\bar{q}\left(x, y, t_{1}\right)-\bar{q}\left(x-u \Delta t, y-v \Delta t, t_{0}\right)\right)^{2} \mathrm{~d} \Omega
$$

Minimizing $\Phi$ to the three unknowns $\bar{q}_{i}^{1}, \frac{\partial q_{i}^{1}}{\partial x}$ and $\frac{\overline{\partial q}_{i}^{1}}{\partial y}$ leads to the relations (7), from which the mean value is updated, and (14) and (15), from which the gradients are updated.

The relations (14) and (15) are worked out as follows:

$$
\begin{aligned}
& \int_{A_{i}}\left\{\bar{q}_{i}^{1}+\frac{\overline{\partial q}_{i}^{1}}{\partial x}\left(x-\bar{x}_{i}\right)+\frac{\overline{\partial q}_{i}^{1}}{\partial y}\left(y-\bar{y}_{i}\right)\right\}\left(x-\bar{x}_{i}\right) \mathrm{d} \Omega= \\
& \int_{A_{i}^{\mathrm{Mat}}}\left\{\bar{q}_{i}^{0}+\frac{\overline{\partial q}_{i}^{0}}{\partial x}\left(x-u_{i} \Delta t-\bar{x}_{i}\right)+\frac{\overline{\partial q}_{i}^{0}}{\partial y}\left(y-v_{i} \Delta t-\bar{y}_{i}\right)\right\}\left(x-\bar{x}_{i}\right) \mathrm{d} \Omega-\sum_{k=1}^{3} D F X_{i, k}^{0} \\
& \int_{A_{i}}\left\{\bar{q}_{i}^{1}+\frac{\overline{\partial q}_{i}^{1}}{\partial x}\left(x-\bar{x}_{i}\right)+\frac{\overline{\partial q}_{i}^{1}}{\partial y}\left(y-\bar{y}_{i}\right)\right\}\left(y-\bar{y}_{i}\right) \mathrm{d} \Omega= \\
& \int_{A_{i}^{\mathrm{Mat}}}\left\{\bar{q}_{i}^{0}+\frac{\overline{\partial q}_{i}^{0}}{\partial x}\left(x-u_{i} \Delta t-\bar{x}_{i}\right)+\frac{\overline{\partial q}_{i}^{0}}{\partial y}\left(y-v_{i} \Delta t-\bar{y}_{i}\right)\right\}\left(y-\bar{y}_{i}\right) \mathrm{d} \Omega-\sum_{k=1}^{3} D F Y_{i, k}^{0}
\end{aligned}
$$

where $A_{i}^{\text {Mat }}$ means that these integrals are calculated over the area where element $i$ would be after a time step $\Delta t$ if the grid points were connected to the material. This situation is represented by the dashed line in Figure 3. Material that is represented with the area $A_{i}$ at $t=t_{0}$, is represented with the area $A_{i}^{\text {Mat }}$ at $t=t_{1}$. The velocities $u_{i}$ and $v_{i}$ are calculated from the nodal point values with the shape functions and determine the shift $\mathbf{v}_{i}^{c} \Delta t$ of the central point of element $i$. The symbols $D F X_{i, k}^{0}$ and $D F Y_{i, k}^{0}$ stand for the following integrals over the shaded part of Figure $2 \mathrm{a}$ or $2 \mathrm{~b}$ dependent on which element the upwind element 


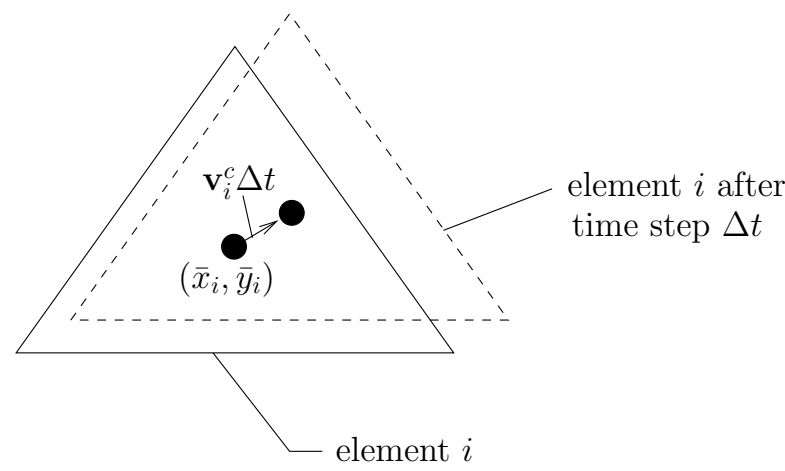

Figure 3: Position of element $i$ after time step $\Delta t$.

is.

$$
\begin{aligned}
& D F X_{i, k}^{0}= \\
& \quad \operatorname{sgn}\left(v_{n, k}\right) \int_{A_{i, k}}\left\{\bar{q}_{\text {up }}^{0}+\frac{\overline{\partial q}_{\text {up }}^{0}}{\partial x}\left(x-u_{\text {up }} \Delta t-\bar{x}_{\text {up }}\right)+\frac{\overline{\partial q}_{\text {up }}^{0}}{\partial y}\left(y-v_{\text {up }} \Delta t-\bar{y}_{\text {up }}\right)\right\}\left(x-\bar{x}_{i}\right) \mathrm{d} \Omega \\
& D F Y_{i, k}^{0}= \\
& \quad \operatorname{sgn}\left(v_{n, k}\right) \int_{A_{i, k}}\left\{\bar{q}_{\text {up }}^{0}+\frac{\overline{\partial q}_{\text {up }}^{0}}{\partial x}\left(x-u_{\text {up }} \Delta t-\bar{x}_{\text {up }}\right)+\frac{\overline{\partial q}_{\text {up }}^{0}}{\partial y}\left(y-v_{\text {up }} \Delta t-\bar{y}_{\text {up }}\right)\right\}\left(y-\bar{y}_{i}\right) \mathrm{d} \Omega
\end{aligned}
$$

More detailed relations of all the integrals in (17) to (20) are given in appendix A.

As already mentioned in section 3.1 linear terms vanish in integrals over a triangle $i$, so that $\bar{q}_{i}^{1}$ vanish in the left hand sides of (17) and (18). Therefore, it can be concluded that $\frac{\overline{\partial q}_{i}^{1}}{\partial x}$ and $\frac{\overline{\partial q}_{i}^{1}}{\partial y}$ are the unknowns.

Since the areas of the shaded parts are dependent on the magnitude of $\left|v_{n, k}\right| \Delta t$, the contribution of a neighbour element is proportional to $\left|v_{n, k}\right|$. In case of small $\left|v_{n, k}\right|, D F X_{i, k}^{0}$ and $D F Y_{i, k}^{0}$ are small (see (19) and (20)), so that (17) and (18) are not influenced very much. Therefore, the derivatives change vanishingly when the convective displacements are small. This is an advantage compared to method I.

\subsection{Limiting}

In order to prevent spurious oscillations limiters are needed in case that method I is used to update the derivatives. For method II it seems that an explicit limiter is redundant (see the results in section 4 ).

When oscillations could arise, derivatives are multiplied by a limiter value. There are several methods to determine a limiter value. Here, we calculate the limiter value $\phi(r)$ 
based on Tamamidis [11]. First, the ratio $r$ is determined, for an edge $k$ given by:

$$
r=\frac{\bar{q}_{\text {down }}^{0}-\bar{q}_{\text {up }}^{0}}{\overline{\delta q}_{\text {up }}^{0}}
$$

where $\bar{q}_{\text {down }}^{0}$ and $\bar{q}_{\text {up }}^{0}$ are the mean values for the downwind and upwind element respectively. The term $\overline{\delta q}_{\text {up }}^{0}$ denotes the higher order terms, given by:

$$
\overline{\delta q}_{\text {up }}^{0}=\frac{\overline{\partial q}_{\text {up }}^{0}}{\partial x}\left(X_{m, k}-\bar{x}_{i}\right)+\frac{\overline{\partial q}_{\text {up }}^{0}}{\partial y}\left(Y_{m, k}-\bar{y}_{i}\right)
$$

where $\left(X_{m, k}, Y_{m, k}\right)$ represents the middle point of side $k$. The value of the scalar function $q$ at this point should lie in the interval between $\bar{q}_{\text {down }}^{0}$ and $\bar{q}_{\text {up }}^{0}$ yielding the following limiter:

$$
\phi(r)=\max (0, \min (r, 1))
$$

In case that method I is used to update the derivatives, the derivatives at the old level $\frac{\overline{\partial q}_{\mathrm{up}}^{0}}{\partial x}$ and $\frac{\overline{\partial q}_{\mathrm{up}}^{0}}{\partial y}$ are multiplied with the limiter $\phi(r)$ in the relations (9) and (13).

The maximum value of the limiter is one, in which case the derivatives are used to the full extent. In extrema, i.e. for negative values of $r$, the limiter is equal to zero, which means that the derivatives are not taken into account. In such cases the second-order accuracy is lost locally.

\section{Results}

\subsection{Molenkamp tests}

In order to check the performance of the convection method the standard two-dimensional Molenkamp test [19] is used. The Molenkamp test is a test problem where a Gaussian profile (see Figure 4) is rotated, so that convection is performed in every direction. The velocity field is described in the nodal points and represents a pure rigid-body rotation:

$$
\begin{aligned}
& u(x, y)=-2 \pi y \\
& v(x, y)=2 \pi x
\end{aligned}
$$

in the domain:

$$
\begin{aligned}
& -1 \leq x \leq 1 \\
& -1 \leq y \leq 1
\end{aligned}
$$

The initial distribution of the scalar field $q$ is a Gaussian distribution and is defined in the integration points:

$$
\begin{aligned}
& q(x, y, 0)=0.01^{4 r^{2}} \\
& \text { with } r=\sqrt{\left(x+\frac{1}{2}\right)^{2}+y^{2}}
\end{aligned}
$$




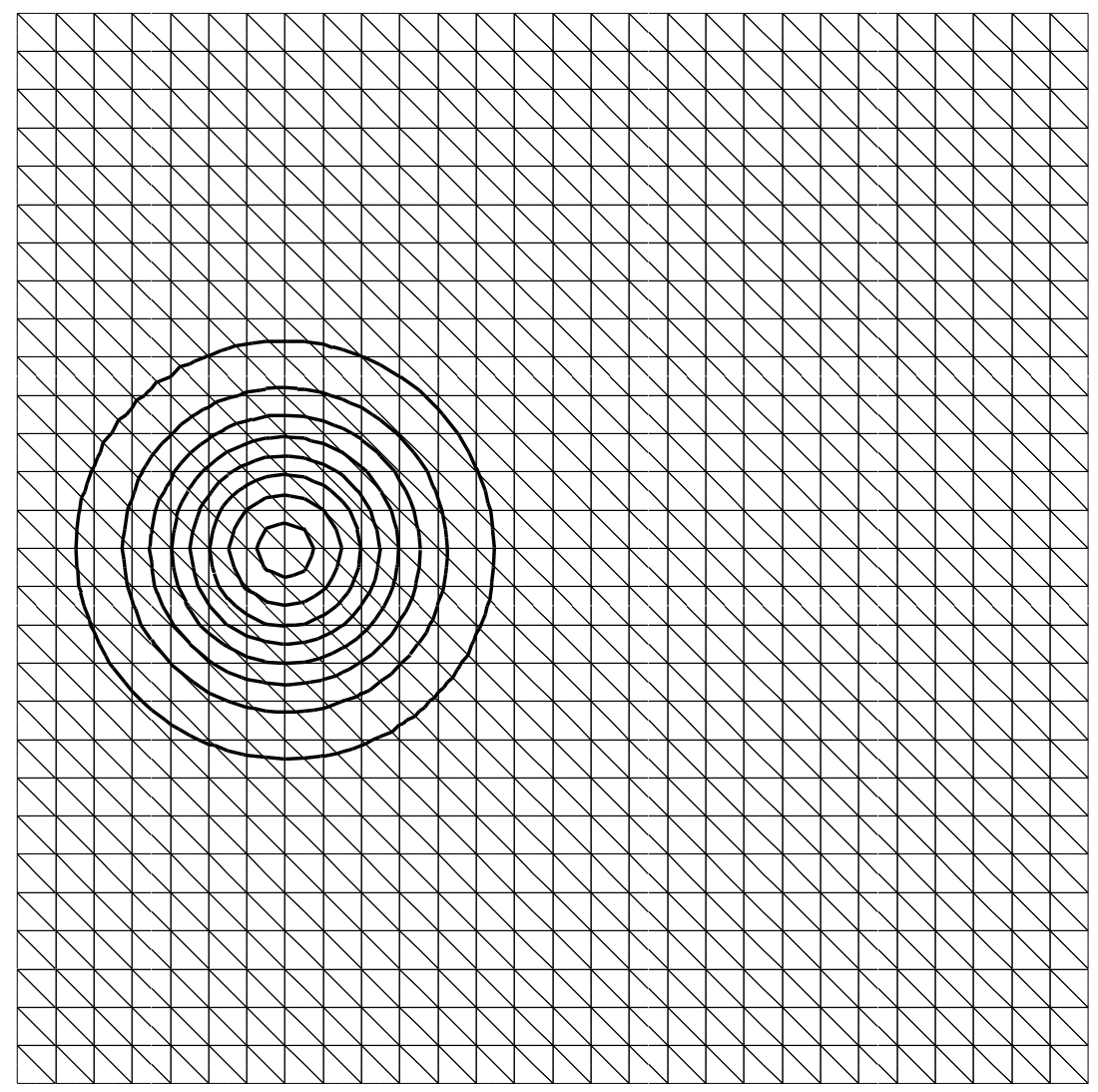

Figure 4: Initial situation for the structured $28 \times 28$ mesh.

Values belonging to the isolines from the outer to the inner one: $0.062 ; 0.188 ; 0.312 ; 0.438 ; 0.562 ; 0.688 ; 0.812 ; 0.938$.

As inflow condition at the boundaries of the domain (25) it is taken that $q$ equals zero.

At $t=1$ one full revolution of the profile is done. At that moment the exact solution $q_{\text {exact }}$ equals the initial situation (26), so that the following two error norms can be determined:

$$
\begin{aligned}
\|\Delta q\|_{1} & =\frac{1}{A} \sum_{i=1}^{N_{\mathrm{el}}} \int_{A_{i}}\left|q-q_{\text {exact }}\right| \mathrm{d} \Omega \\
\|\Delta q\|_{2} & =\sqrt{\frac{1}{A} \sum_{i=1}^{N_{\mathrm{el}}} \int_{A_{i}}\left|q-q_{\text {exact }}\right|^{2} \mathrm{~d} \Omega}
\end{aligned}
$$

where $N_{\mathrm{el}}$ represents the number of elements in the domain and $A$ is the total area of the 
domain, given by:

$$
A=\sum_{i=1}^{N_{\mathrm{el}}} A_{i}
$$

The Molenkamp problem is solved on structured grids as well as unstructured grids of plane strain triangles. In Table 1 some information is given of the used grids. The

\begin{tabular}{|l|c|c|c|l|}
\hline & Grid & $\begin{array}{c}\text { Number of } \\
\text { elements }\end{array}$ & $\begin{array}{c}\text { Number of } \\
\text { nodes }\end{array}$ & Time step \\
\hline \hline Structured & $14 \times 14$ & 392 & 841 & 0.002 \\
& $28 \times 28$ & 1568 & 3249 & 0.001 \\
& $56 \times 56$ & 6272 & 12769 & 0.0005 \\
\hline Unstructured & $14 \times 14$ & 568 & 1193 & 0.00125 \\
& $28 \times 28$ & 2226 & 4565 & 0.000625 \\
& $56 \times 56$ & 8920 & 18065 & 0.0003125 \\
\hline
\end{tabular}

Table 1: Information of the grids.

notation $n_{x} \times n_{y}$ refers to the number of elements on the boundary of the domain, $n_{x}$ in $x$-direction and $n_{y}$ in $y$-direction respectively. For the structured grids quadrilateral elements are created first and after that every quadrangle is divided into two triangles. In this way $2 n_{x} n_{y}$ triangles are constructed for an $n_{x} \times n_{y}$ grid. For the unstructured grids the elements are generated with a mesh generation program that creates a Delaunay-Voronoï mesh [20]. As can be seen in Table 1 the grids are refined with a factor two and four, approximately resulting in four times and sixteen times the number of elements.

The calculations were done with three schemes. First, a First Order Upwind method (FOU) was used where a constant distribution per element is assumed. In this case derivatives are not taken into account and (9) reduces to the simpler form:

$$
F_{i, k}^{0}=\operatorname{sgn}\left(v_{n, k}\right) \int_{A_{i, k}} \bar{q}_{\text {up }}^{0} \mathrm{~d} \Omega=\operatorname{sgn}\left(v_{n, k}\right) A_{i, k} \bar{q}_{\text {up }}^{0}
$$

Method I and method II assume linear distributions per element and the derivatives are updated according to section 3.2.

For every performed calculation $q_{\max }$ and $q_{\min }$ are the maximum and minimum value of the scalar function $q$ evaluated in the integration points. The two norms $\|\Delta q\|_{1}$ and $\|\Delta q\|_{2}$ are determined according to (27) and (28) respectively. The ratios $R_{1}$ and $R_{2}$ are given 
by:

$$
\begin{aligned}
& R_{1}=\frac{\left|\left\|\Delta q_{1}\right\|_{1}-\left\|\Delta q_{2}\right\|_{1}\right|}{||\left|\Delta q_{2}\left\|_{1}-\right\| \Delta q_{4} \|_{1}\right|} \\
& R_{2}=\frac{\left|\left\|\Delta q_{1}\right\|_{2}-\left\|\Delta q_{2}\right\|_{2}\right|}{\left|\left\|\Delta q_{2}\right\|_{2}-\left\|\Delta q_{4}\right\|_{2}\right|}
\end{aligned}
$$

The solution of the $14 \times 14$ grid is represented by $q_{1}$. Whereas $q_{2}$ and $q_{4}$ are the numerical solutions of the $28 \times 28$ and $56 \times 56$ grids respectively. From the ratios $R_{1}$ and $R_{2}$ the convergence rate can be read. By means of Taylor expansion it can be derived that for schemes which are first-order accurate these ratios tend to 2 and that for second-order accuracy these ratios tend to 4 .

Table 2 shows results for structured grids after that one full revolution is done (at $t=1$ ). Method I shows a better convergence rate than the FOU-method, however method

\begin{tabular}{|l|l|l|l|l|l|l|l|}
\hline \multicolumn{10}{|c|}{ Molenkamp test for Structured Grids } \\
\hline \hline \multirow{3}{*}{ Grid } & $q_{\max }$ & $q_{\min }$ & $\|\Delta q\|_{1}$ & $\|\Delta q\|_{2}$ & $R_{1}$ & $R_{2}$ \\
& & & & {$\left[\cdot 10^{-4}\right]$} & {$\left[\cdot 10^{-4}\right]$} & & \\
\hline FOU & $14 \times 14$ & 0.141 & 0.0 & 437.6 & 1189. & & \\
& $28 \times 28$ & 0.251 & 0.0 & 359.8 & 992.6 & & \\
& $56 \times 56$ & 0.400 & 0.0 & 263.4 & 756.4 & 0.81 & 0.83 \\
\hline Method I & $14 \times 14$ & 0.297 & -0.0593 & 289.6 & 927.1 & & \\
& $28 \times 28$ & 0.481 & -0.0268 & 193.8 & 656.7 & & \\
& $56 \times 56$ & 0.641 & $-2.44 \cdot 10^{-3}$ & 122.8 & 429.5 & 1.35 & 1.19 \\
\hline & $14 \times 14$ & 0.796 & -0.0147 & 106.9 & 298.8 & & \\
& $28 \times 28$ & 0.961 & $-1.01 \cdot 10^{-3}$ & 29.22 & 98.90 & & \\
& $56 \times 56$ & 1.02 & $-1.13 \cdot 10^{-7}$ & 14.33 & 51.13 & 5.22 & 4.18 \\
\hline
\end{tabular}

Table 2: Results for the Molenkamp test.

II shows the best convergence rate according to the error norms. The ratios $R_{1}$ and $R_{2}$ are even higher than 4 , so that more than second order accuracy has been reached. Since the solutions of the FOU-method and method I are far away from the exact solution, the ratios $R_{1}$ and $R_{2}$ do not tend to the expected values 2 and 4 respectively. As can be concluded from Table 2 the FOU-method is very diffusive, but the monotonicity requirement is satisfied. Since no gradients have to be updated, the FOU-method is relatively cheap in numerical operations that have to be carried out.

If method I and method II are compared for numerical operations, it is established that method II is the most expensive one. Differences between method I and method II are caused by updating the gradients. The main part of the determination of updated 
gradients is the calculation of integrals. For method II more integrals must be calculated and these integrals are more complicated than the integrals that need to be calculated for method I. Therefore, it can be concluded that method II is more time consuming, but this is compensated by the accuracy.

In Figure 5 the final situation for the $28 \times 28$ mesh, in case that method II has been used, is outlined. Compared with the initial situation (see Figure 4) the situation after one revolution agrees very well.

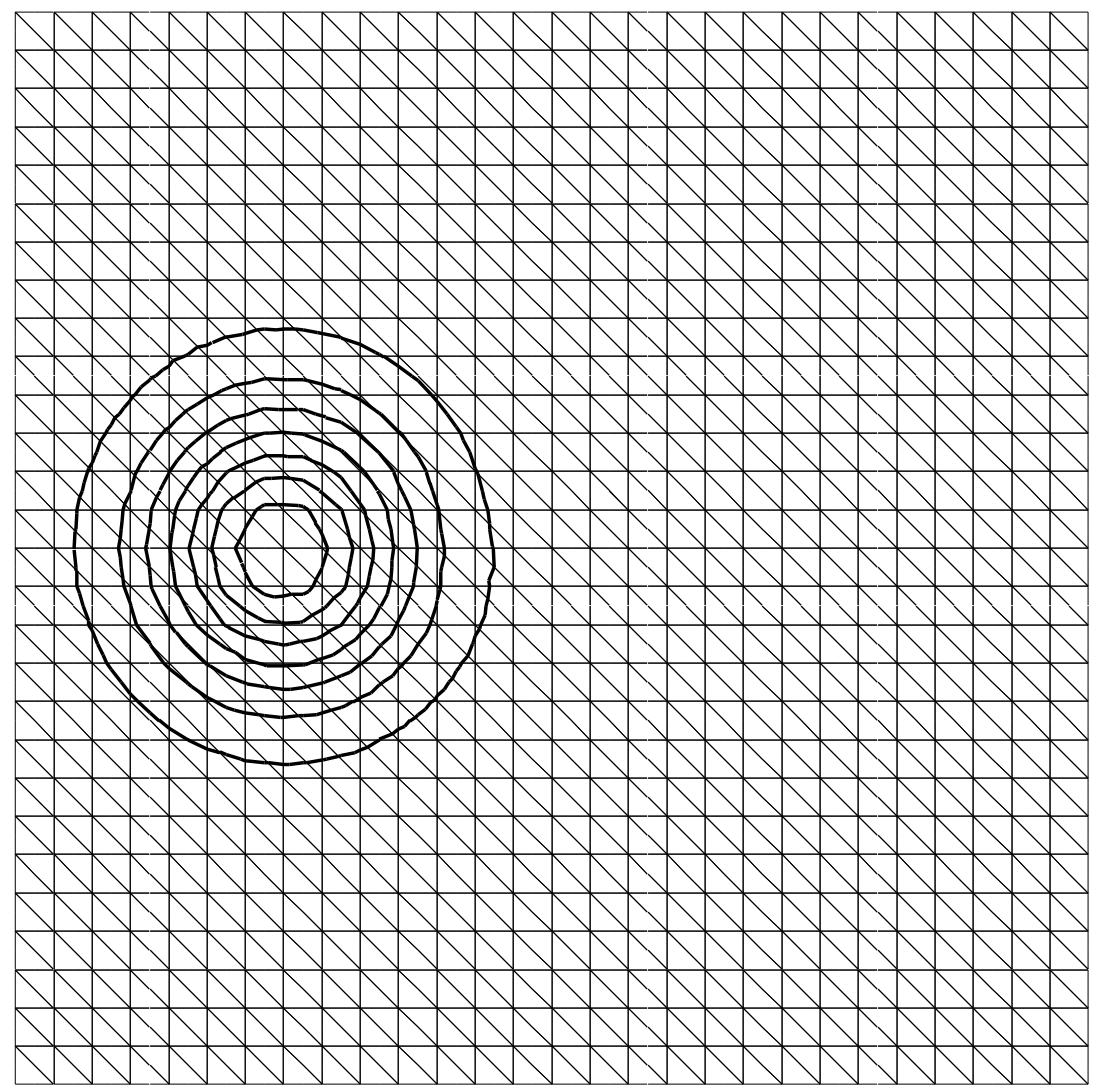

Figure 5: Final situation for the structured $28 \times 28$ mesh using method II.

Values belonging to the isolines from the outer to the inner one: $0.062 ; 0.188 ; 0.312 ; 0.438 ; 0.562 ; 0.688 ; 0.812$.

For unstructured grids the results are shown in Table 3. The results are slightly better than the results for the structured grids. As can be seen in Table 1 the unstructured grids consist of more elements, so that better results could be expected. Besides, the DelaunayVoronoï method strives for grids of equilateral triangles. Since there is not any form of orientation, these elements are known to give better results.

In Figure 6 we show the final situation for the unstructured $28 \times 28$ mesh in case that method II has been used. 


\begin{tabular}{|l|c|c|c|c|c|c|c|}
\hline \multicolumn{10}{|c|}{ Molenkamp test for Unstructured Grids } \\
\hline \hline & Grid & $q_{\max }$ & $q_{\min }$ & $\|\Delta q\|_{1}$ & $\|\Delta q\|_{2}$ & $R_{1}$ & $R_{2}$ \\
& & & & {$\left[\cdot 10^{-4}\right]$} & {$\left[\cdot 10^{-4}\right]$} & & \\
\hline FOU & $14 \times 14$ & 0.184 & 0.0 & 418.5 & 1116. & & \\
& $28 \times 28$ & 0.315 & 0.0 & 323.8 & 888.3 & & \\
& $56 \times 56$ & 0.472 & 0.0 & 227.5 & 656.9 & 0.98 & 0.99 \\
\hline Method I & $14 \times 14$ & 0.448 & -0.0285 & 218.9 & 712.6 & & \\
& $28 \times 28$ & 0.655 & -0.0166 & 123.8 & 425.2 & & \\
& $56 \times 56$ & 0.782 & $-6.08 \cdot 10^{-5}$ & 78.38 & 272.6 & 2.09 & 1.88 \\
\hline Method II & $14 \times 14$ & 0.843 & $-9.15 \cdot 10^{-3}$ & 65.44 & 197.5 & & \\
& $28 \times 28$ & 0.969 & $-1.54 \cdot 10^{-4}$ & 18.19 & 60.88 & & \\
& $56 \times 56$ & 1.02 & $-5.50 \cdot 10^{-5}$ & 10.52 & 39.07 & 6.15 & 6.26 \\
\hline
\end{tabular}

Table 3: Results for the Molenkamp test.

\subsection{Block test}

The Molenkamp tests in the preceding section have shown us that method II gives very accurate results without use of an explicit limiter. In order to perform an extra check on stability, we did a more severe test with the unstructured $28 \times 28$ mesh. This test can be compared with the Molenkamp tests, but instead of a Gaussian profile a block profile is rotated.

The velocity field is described in the nodal points and is given by (24). The domain is also equal to the domain used for the Molenkamp tests and is given by (25). The initial distribution of the scalar field $q$ however is defined as:

$$
q(x, y, 0)= \begin{cases}1 & \text { if }-0.75 \leq x \leq-0.25,-0.25 \leq y \leq 0.25 \\ 0 & \text { otherwise }\end{cases}
$$

The initial distribution is shown in Figure 7. The block is revolved in 1600 time steps of $0.000625 \mathrm{sec}$. At that moment $(t=1 \mathrm{sec})$ the calculated distribution can be compared with the initial situation. In the ideal case these two distributions will coincide.

Only method II was used to do the calculation. In Figure 8 we show the maximum and minimum values during the simulation together with the maximum and minimum values for the exact case. These values are determined from integration point values. After an overshoot of the maximum and an undershoot of the minimum, the maximum and minimum values remain oscillating little around some steady values. These steady values are higher than the exact maximum and lower than the exact minimum. The main conclusion from Figure 8 is that the maximum and minimum value remain bounded. Thus, method II leads to stable results for this severe test. 


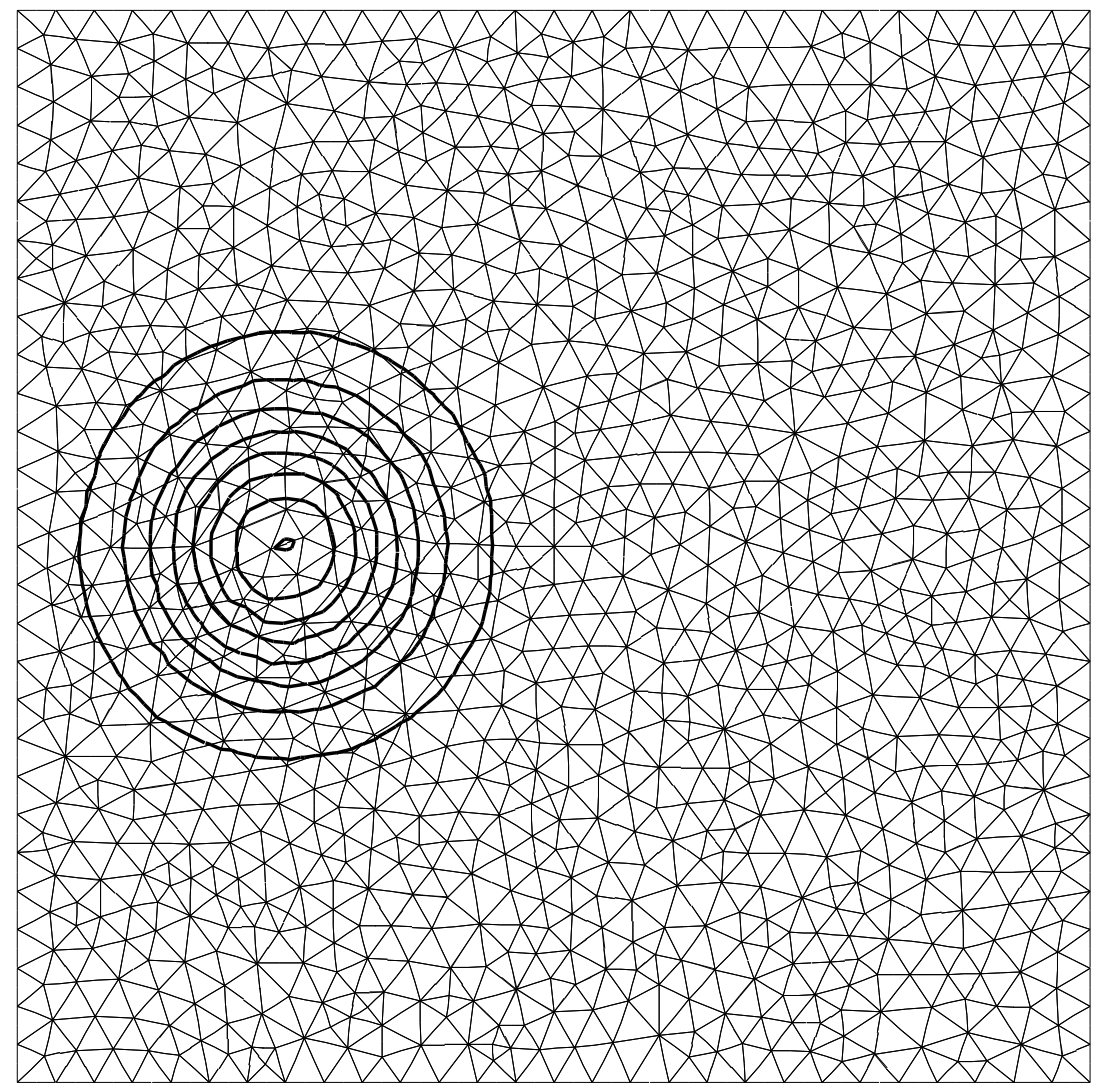

Figure 6: Final situation for the unstructured $28 \times 28$ mesh using method II. Values belonging to the isolines from the outer to the inner one: $0.062 ; 0.188 ; 0.312 ; 0.438 ; 0.562 ; 0.688 ; 0.812 ; 0.938$.

In Figure 9a and 9b the scalar field distributions are shown after an eighth of the revolution and after a full revolution respectively. Comparing these two figures with Figure 7 we see that the block profile is present well after an eighth of the revolution and reasonably well after a full revolution. During the first part of the revolution the scalar field $q$ is smeared out slightly. Comparing Figure $9 \mathrm{a}$ and $9 \mathrm{~b}$ with each other we see that the final distribution is smeared out more than the distribution after an eighth of the revolution.

Similar tests as presented in this section were performed by Tamamidis [11]. A comparison between method II and reference [11] suggests that method II is less diffusive than all methods presented by Tamamidis. It should be noted however that Tamamidis showed results only for structured grids, with slightly different size, different time steps and a cosine instead of a Gauss profile. Moreover the block test was performed with a diagonal translation only and not with the more severe rotation as presented here. 


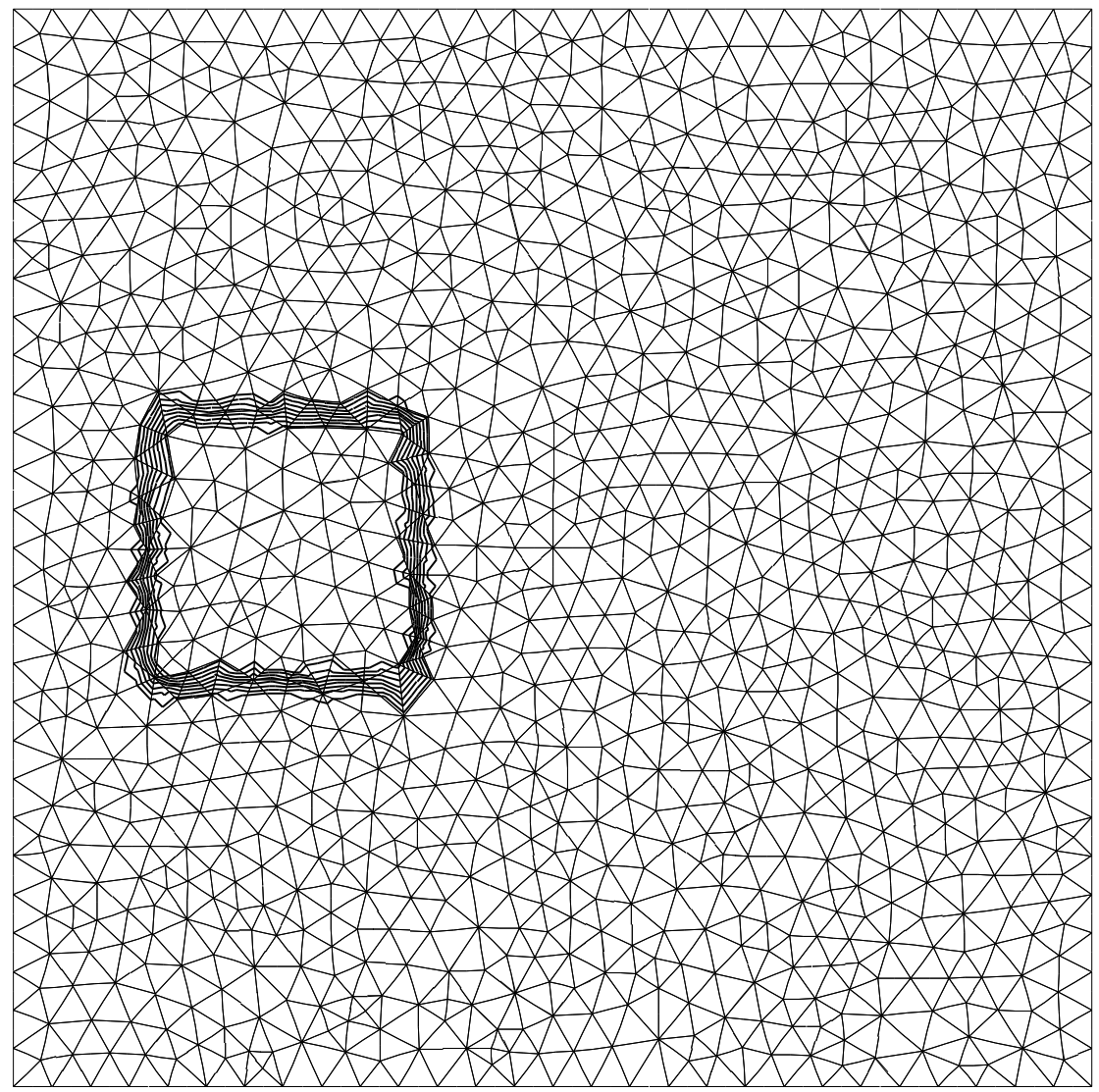

Figure 7: Initial situation for the unstructured $28 \times 28$ mesh. Values belonging to the isolines from the outer to the inner one: $0.062 ; 0.188 ; 0.312 ; 0.438 ; 0.562 ; 0.688 ; 0.812 ; 0.938$.

\section{Extrusion}

In the preceding section the performance of the convection scheme has been tested with the Molenkamp test case as well as with a block problem. In this section the convection scheme is actually applied to the remap in the ALE-method. Since the displacement of the grid points can be chosen independent from the material displacement, some adaptations are necessary to apply the method to an ALE-formulation.

The main difference between solving the convection equation on a fixed grid as discussed in the previous section and the application to an ALE-formulation, is that the area $A_{i}$ of a finite element might change. In the ALE-formulation the initial situation is represented by the situation after the Lagrangian part of the calculation and after one convection step (the Eulerian part of the calculation) the final situation is reached. If the situation before convection and the situation after convection are denoted with 'Lag' and 'Eul' respectively, 


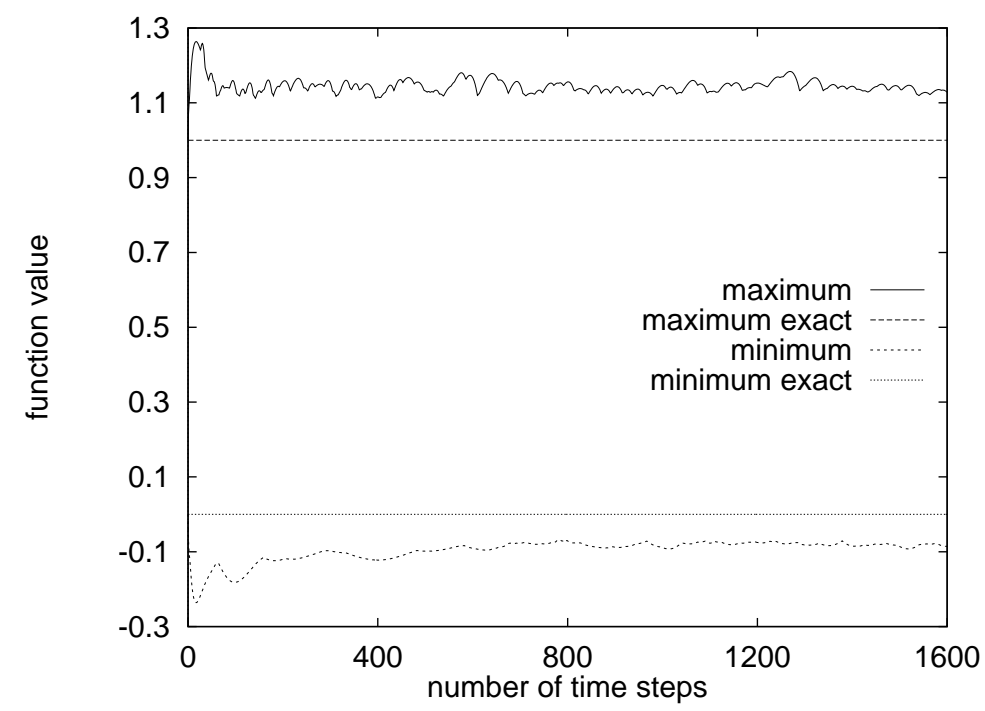

Figure 8: Maximum and minimum values.

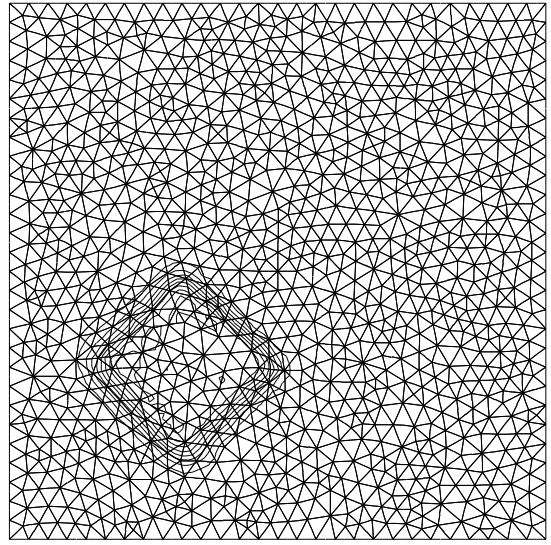

(a)

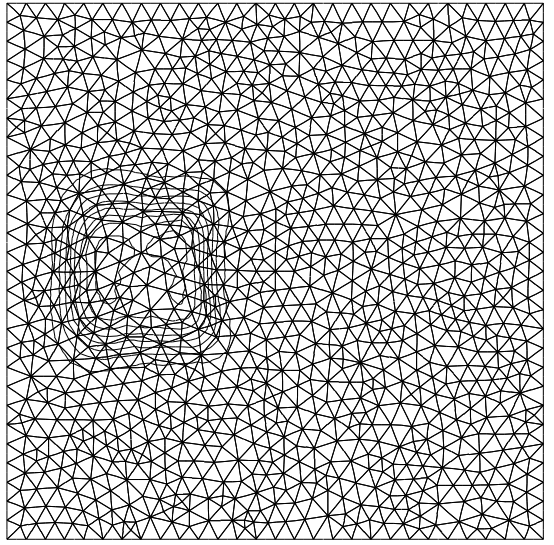

(b)

Figure 9: Scalar field distribution for the unstructured $28 \times 28$ mesh. (a) After an eighth of the revolution. (b) After a full revolution.

Values belonging to the isolines from the outer to the inner one: $0.022 ; 0.167 ; 0.311 ; 0.455$; $0.600 ; 0.744 ; 0.888 ; 1.033$.

the following relation must hold approximately:

$$
A_{i}^{\mathrm{Eul}}=A_{i}^{\mathrm{Lag}}-\operatorname{sgn}\left(v_{n, k}\right) \sum_{k=1}^{3} A_{i, k}
$$

where $v_{n, k}$ is the normal velocity and $A_{i, k}$ is the area of the rectangle as shown in Figure 2.

Another difference is the absence of a real time $\Delta t$. The situation before and after convection are defined at the same point in time. The displacement from the situation 
after the Lagrangian part to the final situation after the Eulerian part is not expressed in the convective velocity $\mathbf{v}^{c}$, but in the convective displacement $\Delta \mathbf{x}^{c}$.

A third item to consider is that some physical quantities, e.g. the equivalent plastic strain, should be monotonically increasing. This is not enforced by the presented algorithm and even negative values could be predicted. The material model can subsequently ignore negative values. This is not used in the presented tests since that would obscure the results.

A simple extrusion process was simulated. A billet was pushed through a die and the thickness was reduced from $4 \mathrm{~mm}$ to $1 \mathrm{~mm}$. We used a rigid-plastic material model, and hardening was described according to the Nadaï relation with the equivalent plastic strain $\varepsilon_{p}$ and yield stress $\sigma_{y}$ related as follows:

$$
\sigma_{y}=C\left(\varepsilon_{0}+\varepsilon_{p}\right)^{n}
$$

where $C=565.32 \mathrm{~N} / \mathrm{mm}^{2}, \varepsilon_{0}=0.23403 \cdot 10^{-10}$ and $n=0.2589$.

The billet was modeled with plane strain quadratic triangles and was discretized $7.5 \mathrm{~mm}$ before the contraction and $7.5 \mathrm{~mm}$ after the contraction. The unstructured mesh as shown in Figure 10 contains 539 elements and there are about 2200 degrees of freedom. The nodal points that lie along the dashed lines were suppressed in perpendicular direction and consequently might displace in tangential direction only. The billet was pushed in 2500 increments to a total displacement of $12.5 \mathrm{~mm}$ at the inlet zone.

In the simulation of the extrusion process the mesh is kept fixed in space. After the Lagrangian part of the calculation the mesh is put back to the initial mesh during the Eulerian part of the calculation. In consequence, the quantities which have to be convected, the equivalent plastic strain, are continuously remapped to the initial mesh. Here, the remap of the plastic strain was performed using a first order upwind method and method II as described in section 3 .

In Figures 11 and 12 the equivalent plastic strain distributions are shown for the first order upwind method and method II respectively. The two plots are based on averaged nodal values. As can be concluded from Figure 11 the equivalent plastic strain distribution in the outlet zone over the thickness is almost homogeneous when the first order upwind method is used. The equivalent plastic strain reduces in streamline direction which is physically unrealistic. On the contrary, method II does not show a homogeneous distribution in the outlet, a gradient over the thickness is distinguished clearly and the equivalent plastic strain does not reduce (see Figure 12). Using a first order method results in much crosswind diffusion, the equivalent plastic strain is smeared out over the thickness after the contraction. Although only a few elements are used over the thickness in the outlet zone, method II convects the plastic strain in the streamline direction very well.

For this simulation we compared the computer time needed for the Eulerian part of the analysis using method II to the time needed for the Lagrangian part. For an implicit Lagrangian step, the most important stages are assembling the stiffness matrix, solving a set of equations and calculating the internal force vector. The CPU time needed for these stages are given in Table 4 . These values show us that the convection of integration 


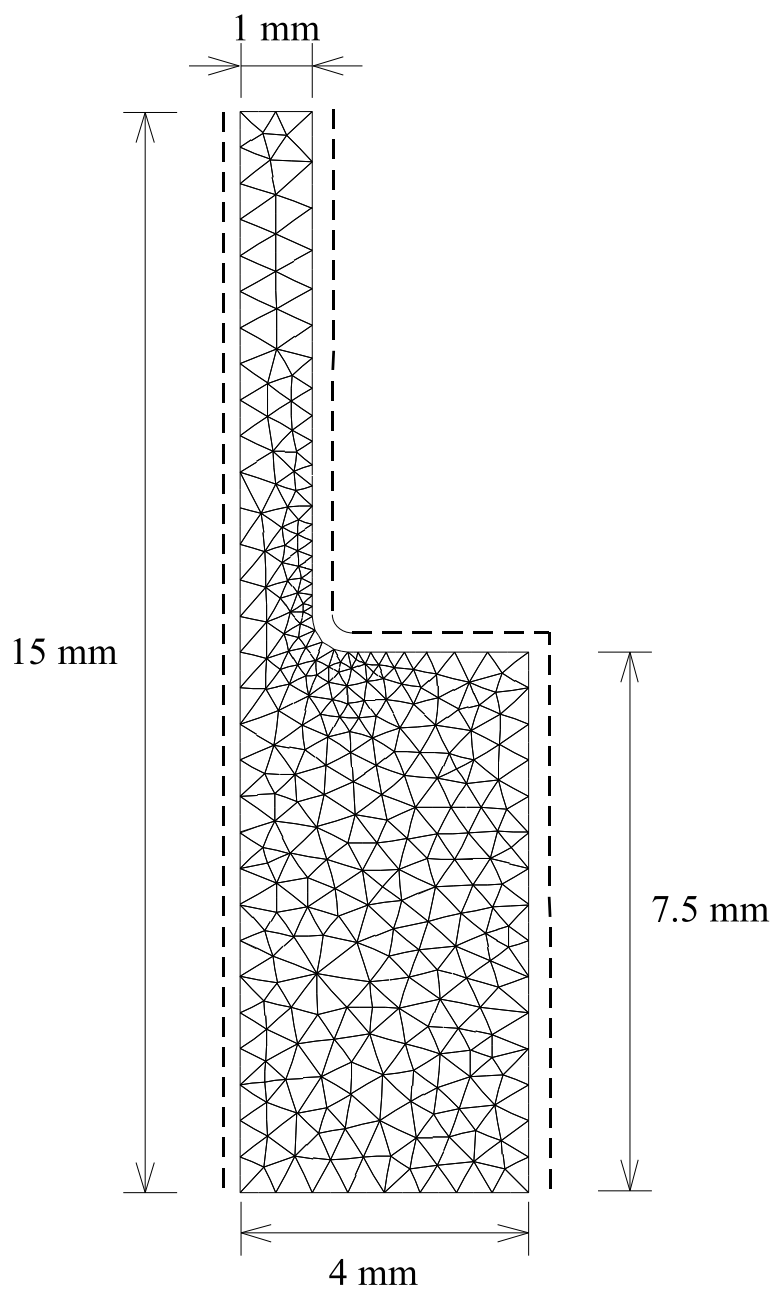

Figure 10: Extrusion process.

\begin{tabular}{|l|r|}
\hline Part of simulation & Time $[\mathrm{msec}]$ \\
\hline \hline Assembly of system matrix & 3000 \\
\hline Solution using direct solver & 57440 \\
\hline Calculation of right hand side & 1840 \\
\hline Convection of integration point values & 2030 \\
\hline
\end{tabular}

Table 4: Computer times of defined parts within one step.

point values takes about 3 percent of the total time used for the other parts. Moreover, the assembly, solve and internal force parts are repeated for every iteration, while the convection part is executed only once per increment. It is clear that the solution stage uses the most computer time. Even if this part can be reduced, e.g. by using more efficient 


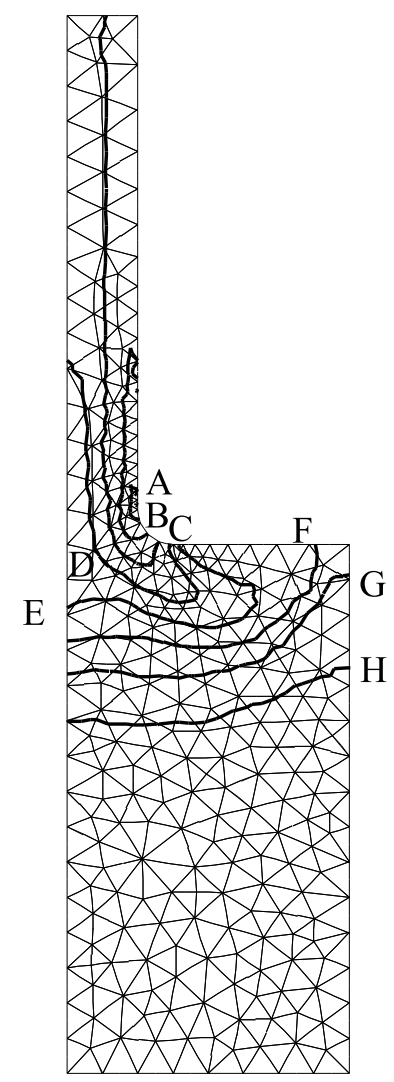

Figure 11: Equivalent plastic strain distribution using a first order upwind method.

Values belonging to the isolines: $A=3.214 ; B=2.786 ; C=2.358$;

$$
D=1.931 ; E=1.503 ; F=1.075 ; G=0.647 ; H=0.220 \text {. }
$$

(iterative) solvers, the time required for the convection algorithm is marginal compared to the Lagrangian step. Therefore, the convective part is not time critical.

Of course, a finer grid with a simple convection algorithm could yield a more efficient Eulerian part, but the finite element calculation where a set of equations has to be solved, will cost much more computer time then. The assembly of the system matrix, the calculation of the internal force vector and the convection algorithm have a linear time complexity (twice the number of elements will take twice as much time), but the time needed for the solver increases faster (typically proportional to $n^{2}$ for $2 \mathrm{D}$ and $n^{7 / 3}$ for $3 \mathrm{D}$ with direct solvers). Therefore, within the framework of ALE-simulations the accuracy of the convection scheme weighs more heavily than the efficiency. 


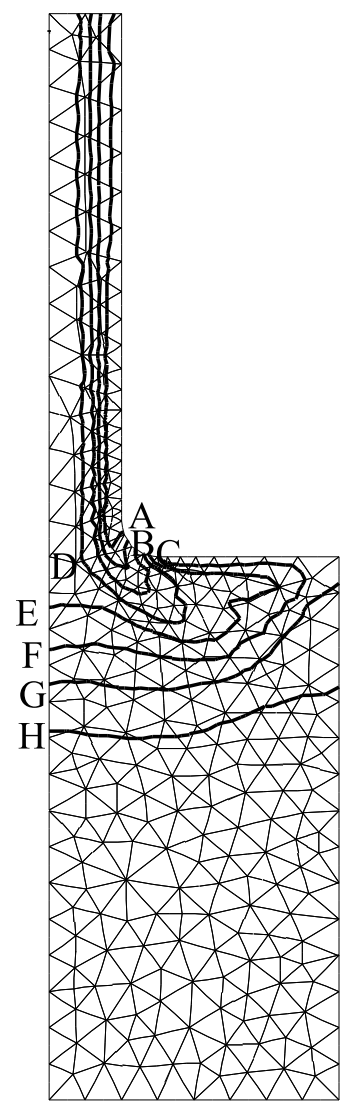

Figure 12: Equivalent plastic strain distribution using method II. Values belonging to the isolines: $A=3.214 ; B=2.786 ; C=2.358$; $D=1.931 ; E=1.503 ; F=1.075 ; G=0.647 ; H=0.220$.

\section{Conclusions}

The presented convection method II shows very accurate results for the Molenkamp tests as well as the block test. The scheme is stable without use of a limiter. The absence of a limiter is an advantage. Usually, the calculation of limiter values costs moderate computer time.

In this scheme no direct time discretization is done. The distance $\mathbf{v}^{c} \Delta t$ determines the shift of the approximate distribution $\bar{q}\left(x, y, t_{0}\right)$. The rectangles in Figure 2 represent an approximation for the real information, represented by trapeziums, that is convected 'through' an edge of the triangle. In case of large values of $\mathbf{v}^{c} \Delta t$ the rectangle becomes a bad approximation. Repeating the Molenkamp tests and the block test for twice as large time steps, leads to an increase of the error norms and a lower convergence rate, but the results remain stable. 
The extrusion simulation has shown that the new convection scheme can be applied to ALE-formulations. In comparison with other parts that must be performed during one step of an ALE-simulation, the convection part takes marginal computer time. The scheme convects local gradients very well and they are still present after a lot of steps. The first order upwind method however is not able to deal with sharp gradients and information is smeared out.

Finally, we remark that the application of the integration point based convection scheme as discussed in this article is not limited to quadratic triangular elements. The principle can be applied to other kinds of two-dimensional elements, but also to three-dimensional elements. In three-dimensional cases a gradient in $z$-direction has to be added, and for the determination of updated gradients the first moment along the $z$-axis has to be dealt with beside the first moments along the $x$ - and $y$-axis (see (14) and (15)).

\section{A Integrals for the new convection scheme}

In this appendix integrals used in the new convection scheme for triangular elements (see section 3) are worked out. These integrals can be split into integrals over triangles and integrals over rectangles. For convenience the integrals are worked out in sums over the corner nodes. In these sums the index $j$ refers to the node number. As the reader will observe, $j-1$ can become 0 . In that case $j-1$ represents node 3 for a triangle and node 4 for a rectangle. The reader will also observe that $j+1$ exceeds the maximum node number. In case that $j+1$ reaches the value 4 for a triangle and the value 5 for a rectangle, $j+1$ represents node 1 .

The flux as given in (9) is an integral over a rectangle as shown in Figure 13 and is given by:

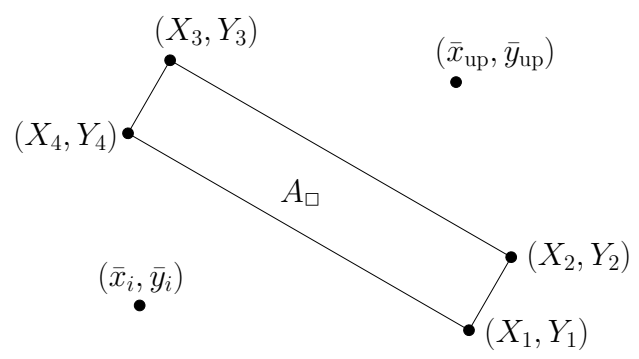

Figure 13: Arbitrary rectangle. 


$$
\begin{aligned}
\int_{A_{\square}}\left\{\bar{q}_{\mathrm{up}}^{0}+\frac{\overline{\partial q}_{\mathrm{up}}^{0}}{\partial x}\left(x-\bar{x}_{\mathrm{up}}\right)+\frac{\overline{\partial q}_{\mathrm{up}}^{0}}{\partial y}\left(y-\bar{y}_{\mathrm{up}}\right)\right\} \mathrm{d} \Omega= \\
A_{\square}\left(\bar{q}_{\mathrm{up}}^{0}-\frac{\overline{\partial q}_{\mathrm{up}}^{0}}{\partial x} \bar{x}_{\mathrm{up}}-\frac{\overline{\partial q}_{\mathrm{up}}^{0}}{\partial y} \bar{y}_{\mathrm{up}}\right)+ \\
\frac{1}{6} \frac{\overline{\partial q}_{\mathrm{up}}^{0}}{\partial x} \sum_{j=1}^{4} X_{j}\left(X_{j}\left(Y_{j+1}-Y_{j-1}\right)-Y_{j}\left(X_{j+1}-X_{j-1}\right)\right)+ \\
\frac{1}{6} \frac{\overline{\partial q}_{\mathrm{up}}^{0}}{\partial y} \sum_{j=1}^{4} Y_{j}\left(X_{j}\left(Y_{j+1}-Y_{j-1}\right)-Y_{j}\left(X_{j+1}-X_{j-1}\right)\right)
\end{aligned}
$$

With:

$$
A_{\square}=\frac{1}{2} \sum_{j=1}^{4} X_{j}\left(Y_{j+1}-Y_{j-1}\right)
$$

representing the area of the rectangle.

Remark that the elaborated relation also hold for an arbitrary quadrilateral.

The left hand sides of (17) and (18) are integrals over a triangle as shown in Figure 14 and yield:

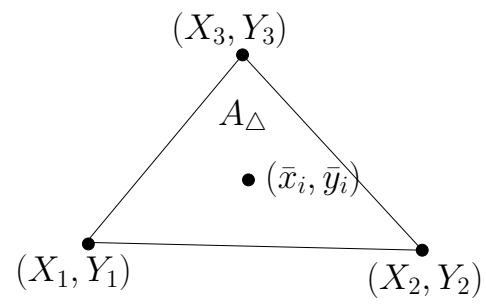

Figure 14: Arbitrary triangle.

$$
\begin{gathered}
\int_{A_{\triangle}}\left\{\bar{q}_{i}^{1}+\frac{\overline{\partial q}_{i}^{1}}{\partial x}\left(x-\bar{x}_{i}\right)+\frac{\overline{\partial q}_{i}^{1}}{\partial y}\left(y-\bar{y}_{i}\right)\right\}\left(x-\bar{x}_{i}\right) \mathrm{d} \Omega= \\
\frac{1}{36} A_{\triangle}\left(\frac{\overline{\partial q}_{i}^{1}}{\partial x} \sum_{j=1}^{3} X_{j}\left(2 X_{j}-X_{j+1}-X_{j-1}\right)+\right. \\
\left.\frac{\overline{\partial q}_{i}^{1}}{\partial y} \sum_{j=1}^{3} X_{j}\left(2 Y_{j}-Y_{j+1}-Y_{j-1}\right)\right)
\end{gathered}
$$




$$
\begin{gathered}
\int_{A_{\triangle}}\left\{\bar{q}_{i}^{1}+\frac{\overline{\partial q}_{i}^{1}}{\partial x}\left(x-\bar{x}_{i}\right)+\frac{\overline{\partial q}_{i}^{1}}{\partial y}\left(y-\bar{y}_{i}\right)\right\}\left(y-\bar{y}_{i}\right) \mathrm{d} \Omega= \\
\frac{1}{36} A_{\triangle}\left(\frac{\overline{\partial q}_{i}^{1}}{\partial x} \sum_{j=1}^{3} Y_{j}\left(2 X_{j}-X_{j+1}-X_{j-1}\right)+\right. \\
\left.\frac{\overline{\partial q}_{i}^{1}}{\partial y} \sum_{j=1}^{3} Y_{j}\left(2 Y_{j}-Y_{j+1}-Y_{j-1}\right)\right)
\end{gathered}
$$

In (38) and (39) it is processed that $\bar{x}_{i}=\frac{1}{3} \sum_{j=1}^{3} X_{j}$ and $\bar{y}_{i}=\frac{1}{3} \sum_{j=1}^{3} Y_{j}$. The area of the triangle is given by:

$$
A_{\triangle}=\frac{1}{2} \sum_{j=1}^{3} X_{j}\left(Y_{j+1}-Y_{j-1}\right)
$$

The integrals over $A_{i}^{\text {Mat }}$ in the right hand sides of (17) and (18) are similar to the previous two integrals (38) and (39). An important difference is that the convective velocity must be taken into account to process the shift of the central point $\left(\bar{x}_{i}, \bar{y}_{i}\right)$. This leads to the following two relations:

$$
\begin{aligned}
\int_{A_{\triangle}}\left\{\bar{q}_{i}^{0}+\frac{\overline{\partial q}_{i}^{0}}{\partial x}\left(x-u_{i} \Delta t-\bar{x}_{i}\right)+\frac{\overline{\partial q}_{i}^{0}}{\partial y}\left(y-v_{i} \Delta t-\bar{y}_{i}\right)\right\}\left(x-\bar{x}_{i}\right) \mathrm{d} \Omega= \\
A_{\triangle}\left\{\frac{1}{3} \bar{q}_{i}^{0}\left(\sum_{j=1}^{3} X_{j}-3 \bar{x}_{i}\right)+\right. \\
\frac{1}{12} \frac{\overline{\partial q}_{i}^{0}}{\partial x}\left(\sum_{j=1}^{3} X_{j}\left(2 X_{j}+X_{j+1}+X_{j-1}-4 u_{i} \Delta t-8 \bar{x}_{i}\right)+12 \bar{x}_{i}\left(u_{i} \Delta t+\bar{x}_{i}\right)\right)+ \\
\frac{1}{12} \frac{\overline{\partial q}_{i}^{0}}{\partial y}\left(\sum_{j=1}^{3}\left(Y_{j}\left(2 X_{j}+X_{j+1}+X_{j-1}-4 \bar{x}_{i}\right)-4 X_{j}\left(v_{i} \Delta t+\bar{y}_{i}\right)\right)+\right. \\
\left.\left.12 \bar{x}_{i}\left(v_{i} \Delta t+\bar{y}_{i}\right)\right)\right\}
\end{aligned}
$$




$$
\begin{aligned}
\int_{A_{\triangle}}\left\{\bar{q}_{i}^{0}+\frac{\overline{\partial q}_{i}^{0}}{\partial x}\left(x-u_{i} \Delta t-\bar{x}_{i}\right)+\frac{\overline{\partial q}_{i}^{0}}{\partial y}\left(y-v_{i} \Delta t-\bar{y}_{i}\right)\right\}\left(y-\bar{y}_{i}\right) \mathrm{d} \Omega= \\
2 A_{\triangle}\left\{\frac{1}{3} \bar{q}_{i}^{0}\left(\sum_{j=1}^{3} Y_{j}-3 \bar{y}_{i}\right)+\right. \\
\frac{1}{12} \frac{\overline{\partial q}_{i}^{0}}{\partial x}\left(\sum_{j=1}^{3}\left(X_{j}\left(2 Y_{j}+Y_{j+1}+Y_{j-1}-4 \bar{y}_{i}\right)-4 Y_{j}\left(u_{i} \Delta t+\bar{x}_{i}\right)\right)+\right. \\
\frac{1}{12} \frac{\overline{\partial q}_{i}^{0}}{\partial y}\left(\sum_{j=1}^{3} Y_{j}\left(u_{i} \Delta t+\bar{x}_{i}\right)\right)+
\end{aligned}
$$

Finally, expressions for the integrals in (19) and (20) are given (see Figure 13):

$$
\begin{aligned}
& \left.\int_{A_{\square}}\left\{\bar{q}_{\text {up }}^{0}+\frac{{\overline{\partial q_{\text {up }}}}_{\partial x}}{\partial x}-u_{\text {up }} \Delta t-\bar{x}_{\text {up }}\right)+\frac{\overline{\partial q}_{\text {up }}^{0}}{\partial y}\left(y-v_{\text {up }} \Delta t-\bar{y}_{\text {up }}\right)\right\}\left(x-\bar{x}_{i}\right) \mathrm{d} \Omega=
\end{aligned}
$$

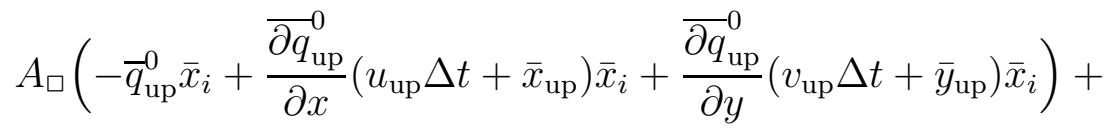

$$
\begin{aligned}
& \frac{1}{6} \bar{q}_{\text {up }}^{0} \sum_{j=1}^{4} X_{j}\left(X_{j}\left(Y_{j+1}-Y_{j-1}\right)-Y_{j}\left(X_{j+1}-X_{j-1}\right)\right)+ \\
& \frac{1}{12} \frac{\overline{\partial q}_{\text {up }}^{0}}{\partial x} \sum_{j=1}^{4} X_{j}\left\{X _ { j } \left(X_{j+1} Y_{j+1}-X_{j-1} Y_{j-1}+\right.\right. \\
& X_{j}\left(X_{j}-2\left(u_{\mathrm{up}} \Delta t+\bar{x}_{i}+\bar{x}_{\mathrm{up}}\right)\right)\left(Y_{j+1}-Y_{j-1}\right)- \\
& \left.Y_{j}\left(X_{j}-2\left(u_{\mathrm{up}} \Delta t+\bar{x}_{i}+\bar{x}_{\mathrm{up}}\right)\right)\left(X_{j+1}-X_{j-1}\right)\right\}+ \\
& \frac{1}{24} \frac{\overline{\partial q}_{\mathrm{up}}^{0}}{\partial y} \sum_{j=1}^{4} X_{j}\left\{X_{j}\left(Y_{j+1}^{2}-Y_{j-1}^{2}\right)+\right. \\
& 2 Y_{j}\left(X_{j}-2 \bar{x}_{i}-2\left(v_{\mathrm{up}} \Delta t+\bar{y}_{\mathrm{up}}\right) \frac{X_{j}}{Y_{j}}\right)\left(Y_{j+1}-Y_{j-1}\right)- \\
& \left.2 Y_{j}\left(Y_{j}-2 \bar{x}_{i} \frac{Y_{j}}{X_{j}}-2\left(v_{\mathrm{up}} \Delta t+\bar{y}_{\mathrm{up}}\right)\right)\left(X_{j+1}-X_{j-1}\right)\right\}
\end{aligned}
$$




$$
\begin{gathered}
\int_{A_{\square}}\left\{\bar{q}_{\mathrm{up}}^{0}+\frac{\overline{\partial q}_{\mathrm{up}}^{0}}{\partial x}\left(x-u_{\mathrm{up}} \Delta t-\bar{x}_{\mathrm{up}}\right)+\frac{\overline{\mathrm{q}}_{\mathrm{up}}^{0}}{\partial y}\left(y-v_{\mathrm{up}} \Delta t-\bar{y}_{\mathrm{up}}\right)\right\}\left(y-\bar{y}_{i}\right) \mathrm{d} \Omega= \\
A_{\square}\left(-\bar{q}_{\mathrm{up}}^{0} \bar{y}_{i}+\frac{\overline{\partial q}_{\mathrm{up}}^{0}}{\partial x}\left(u_{\mathrm{up}} \Delta t+\bar{x}_{\mathrm{up}}\right) \bar{y}_{i}+\frac{\overline{\mathrm{up}}_{\partial y}}{\partial y}\left(v_{\mathrm{up}} \Delta t+\bar{y}_{\mathrm{up}}\right) \bar{y}_{i}\right)+ \\
\frac{1}{6} \bar{q}_{\mathrm{up}}^{0} \sum_{j=1}^{4} Y_{j}\left(X_{j}\left(Y_{j+1}-Y_{j-1}\right)-Y_{j}\left(X_{j+1}-X_{j-1}\right)\right)+ \\
\frac{1}{24} \frac{\overline{\partial q}_{\mathrm{up}}^{0} \sum_{j=1}^{4} Y_{j}\left\{-Y_{j}\left(X_{j+1}^{2}-X_{j-1}^{2}\right)+\right.}{2 X_{j}\left(X_{j}-2 \bar{y}_{i} \frac{X_{j}}{Y_{j}}-2\left(u_{\mathrm{up}} \Delta t+\bar{x}_{\mathrm{up}}\right)\right)\left(Y_{j+1}-Y_{j-1}\right)-} \\
\left.2 X_{j}\left(Y_{j}-2 \bar{y}_{i}-2\left(u_{\mathrm{up}} \Delta t+\bar{x}_{\mathrm{up}}\right) \frac{Y_{j}}{X_{j}}\right)\left(X_{j+1}-X_{j-1}\right)\right\}+ \\
\frac{1}{12} \frac{\overline{\partial q}_{\mathrm{up}}^{0} \sum_{j=1}^{4} Y_{j}\left\{-Y_{j}\left(X_{j+1} Y_{j+1}-X_{j-1} Y_{j-1}\right)+\right.}{X_{j}\left(Y_{j}-2\left(v_{\mathrm{up}} \Delta t+\bar{y}_{i}+\bar{y}_{\mathrm{up}}\right)\right)\left(Y_{j+1}-Y_{j-1}\right)-} \\
\left.Y_{j}\left(Y_{j}-2\left(v_{\mathrm{up}} \Delta t+\bar{y}_{i}+\bar{y}_{\mathrm{up}}\right)\right)\left(X_{j+1}-X_{j-1}\right)\right\}
\end{gathered}
$$

Remark that the last two elaborated relations also hold for an arbitrary quadrilateral.

\section{References}

[1] B. van Leer. Towards the ultimate conservative difference scheme IV. A new approach to numerical convection. J. Comput. Phys., 23:276-299, 1977.

[2] W. K. Liu, H. Chang, J. S. Chen, and T. Belytschko. Arbitrary Lagrangian-Eulerian Petrov-Galerkin finite elements for nonlinear continua. Comput. Methods Appl. Mech. Engrg., 68:259-310, 1988.

[3] D. J. Benson. An efficient, accurate, simple ALE method for nonlinear finite element programs. Comput. Methods Appl. Mech. Engrg., 72:305-350, 1989.

[4] F. P. T. Baaijens. An U-ALE formulation of 3-D unsteady viscoelastic flow. Int. $j$. numer. methods eng., 36:1115-1143, 1993.

[5] J. Huétink. On the simulation of thermomechanical forming processes. PhD thesis, University of Twente, Enschede, 1986. 
[6] J. Donea. Arbitrary Lagrangian-Eulerian finite element methods. In T. Belytschko and T. J. R. Hughes, editors, Computer Methods in Mechanics: Vol 1. Computer Methods for Transient Analysis, volume 1, pages 473-516, Amsterdam, 1983. NorthHolland.

[7] T. J. R. Hughes, W. K. Liu, and T. K. Zimmerman. Lagrangian-Eulerian finite element formulation. Comput. Methods Appl. Mech. Engrg., 29:329-349, 1981.

[8] A. Huerta, F. Casadei, and J. Donea. ALE stress update in transient plasticity problems. In D. R. J. Owen and E. Oñate, editors, Computational Plasticity: Fundamentals and Applications, pages 1865-1876. Pineridge Press, 1995.

[9] F. P. T. Baaijens. Numerical experiments with a discontinous Galerkin method including monotonicity enforcement on the stick-slip problem. J. Non-Newtonian Fluid Mechanics, 51:141-159, 1994.

[10] C. Stoker, C. Gay, F. Bay, and J.-L. Chenot. A velocity approach for the ALEmethod applied to 2D and 3D problems. In J. Huétink and F. P. T. Baaijens, editors, Simulation of Materials Processing: Theory, Methods and Applications, pages 95-101. Balkema Rotterdam, 1998.

[11] P. Tamamidis. A new upwind scheme on triangular meshes using the finite volume method. Comput. Methods Appl. Mech. Engrg., 124:15-31, 1995.

[12] C. J. Hwang and S. J. Wu. Adaptive finite volume upwind approach on mixed quadrilateral-triangular meshes. AIAA J., 31:61-67, 1993.

[13] P. N. van der Helm, J. Huétink, and R. Akkerman. Comparison of artificial dissipation and limited flux schemes in Arbitrary Lagrangian-Eulerian finite element formulations. Int. j. numer. methods eng., 41:1057-1076, 1998.

[14] G. Rekers. Numerical simulations of unsteady viscoelastic flow of polymers. $\mathrm{PhD}$ thesis, University of Twente, Enschede, 1995.

[15] M. J. van Haaren. ALE-simulations with quadratic triangular elements. Postgraduate report, University of Twente, Enschede, 1998.

[16] H. C. Stoker. Developments of the Arbitrary Lagrangian-Eulerian Method in nonlinear Solid Mechanics. Applications to Forming Processes. PhD thesis, University of Twente, Enschede, 1999.

[17] J. P. Ponthot. Efficient mesh management in Eulerian-Lagrangian method for large deformation analysis. In Proceedings of the $3^{\text {rd }}$ international conference on numerical methods in industrial forming processes, pages 203-210. Balkema Rotterdam, 1989.

[18] S. D. Conte. Elementary Numerical Analysis: An Algorithmic Approach. McGrawHill, New York, 1965. 
[19] C. B. Vreugdenhil and B. Koren, editors. Numerical Methods for Advection-Diffusion Problems, volume 45: Notes on Numerical Fluid Mechanics. Braunschweig, Vieweg, 1993.

[20] P. L. George. Automatic Mesh Generation, Application to Finite Element Methods. John Wiley \& Sons, Masson, 1991. 